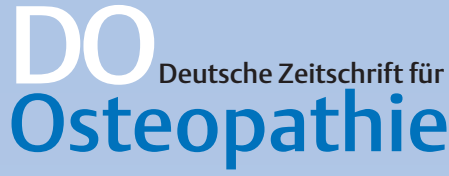

Offizielles Organ folgender Verbände

Verband der Osteopathen Deutschland e.V. (VOD)

Deutsche Akademie für Osteopathische Medizin e.V. (DAOM)

Dssociation Luxembourgeoise des Ostéopathes (A.L.D.O.)

ISSN 1610-5044

$3 / 2005$, 3. Jahrgang

Herausgeber:

Prof. Dr. rer. nat. med. habil. Rainer Breul D.O.h.c.

Marina Fuhrmann D.O. M.R.O.

Prof. Dr. med. habil. Karl-Ludwig Resch

Dr. med. Roger Seider D.O.

\section{Redaktioneller Beirat:}

Jo Buekens, Osteopath D.O., College Sutherland, Garnich,

Luxemburg

Prof. Dr. med. Jochen Fanghänel, Institut für Anatomie,

Ernst-Moritz-Arndt-Universität, Greifswald

Dr. med. Edgar Hinkelthein, Osteopath D.O., M.R.O., B. Sc. (Hons.)

Osteopathieschule Damp, Fleckeby

Werner Langer, Osteopath D.O., IfAO - Institut fü

Angewandte Osteopathie, Bitburg

Dr. med. Horst P. Schwerdtner, DAOM, Hamm

Wissenschaftlicher Beirat:

Prof. Dr. med. Jan Cabri, Centro de Est. de Fisioterapie, Faculdade

de Motricidade Hum., Lissabon, Portugal

Prof. Anthony G. Chila D.O. FAAO, College of Osteopathic

Medicine, Ohio University, Athens, Ohio USA

Christian Fossum D.O., European School of Osteopathy (ESO),

Maidstone, England

Dr. Viola M. Frymann D.O. FAAO, FCA;

Director of the Osteopathic Center for Children, San Diego,

Kalifornien USA

Prof. Dr UA Karin Kraft, Lehrstuhl für Naturheilkunde,

Universität Rostock

Renzo Molinari, Principal of the European School for Osteopathy

(ESO), Maidstone, England

Dr. med. Heike Philippi, Kinderneurologin, Schlangenbad

Prof. Frank H. Willard Ph. D., College of Osteopathic Medicine,

University of New England, Biddeford, ME USA

Verlag

Hippokrates Verlag in

MVS Medizinverlage Stuttgart GmbH \& Co.KG

Oswald-Hesse-Str. 50, 70469 Stuttgart

Redaktion (v.i.S.d.P.)

Marina Horbatsch

Fon/Fax: (0711) 8931-715/705

E-Mail: marina.horbatsch@medizinverlage.de

Christoph Newiger in team $4 \mathrm{U}$ Medienbüro

Widenmayerstr. 42, 80538 München

Fon/Fax: (089) 20 9001-67/-68

E-Mail: redaktion.do@medizinverlage.de

Marketing

Marion Krubasik

Fon/Fax: (0711) 8931-735/748

E-Mail: marion.krubasik@medizinverlage.de

Anzeigen:

Achim Wienert, MVS Mediaservice

Fon/Fax: (07 11) 8931-734/-706

E-Mail: achim.wienert@medizinverlage.de

Abonnementservice:

Fon/Fax: (07 11) 8931-321/-422

E-Mail: aboservice@thieme.de

Probeabonnement:

Fon/Fax: (07 11) 8931-906/-901

E-Mail: kundenservice@thieme.de

Produktion:

Satz \& Repro: Fotosatz Sauter, Donzdorf

Druck \& Bindung: Rondo Druck, Ebersbach

Autorenhinweise:

Auf Anfrage bei der Redaktion.

Erscheinungsweise:

Vierteljährlich

Bezugspreise 2005:

$\begin{array}{llll} & \begin{array}{l}\text { Abonnement- } \\ \text { preis }\end{array} & \begin{array}{l}\text { Versand- } \\ \text { kosten }\end{array} & \text { Gesamt } \\ \text { Inland } & € 67,90 & € 7,20 & € 75,10 \\ \text { Europa } & € 67,90 & € 12,90 & € 80,80 \\ \text { Restl. Welt } & € 67,90 & € 21,50 & € 89,40\end{array}$

Aus-, Fort- und Weiterbildung, Studenten:

$\begin{array}{llll}\text { Inland } & € 41,90 & € 7,20 & € 49,10 \\ \text { Europa } & € 41,90 & € 12,90 & € 54,80 \\ \text { Restl. Welt } & € 41,90 & € 21,50 & € 63,40\end{array}$

Fortsetzung auf S.2

\title{
Osteopathie und Spiritualität
}

G oethes Faust („Habe nun, ach! Philosophie, Juristerei und Medizin, und leider auch Theologie durchaus studiert, mit heißem Bemühn.") hätte sich durchaus professionell kompetent fühlen können, hätte er sich neben Körper (Juristerei und Medizin) und Geist (Philosophie) auch um die Seele kümmern wollen.

Bis heute aber trennt ein bemerkenswerter akademischer Graben gerade Medizin („Naturwissenschaft“) und Spiritualität („Theologie“). Auch wenn viele Menschen, die den Zugang zu den Kirchen (und damit zu den Theologen) verloren haben, gerade und vor allem bei Angehörigen medizinischer Fachberufe auch spirituellen Beistand suchen.

Dessen ungeachtet beschränken sich weiterhin die physiotherapeutische Ausbildung auf das Physische, die psychotherapeutische auf das Psychische und die ärztliche gar darauf, die spirituelle Ebene als jenseits der eigenen Zuständigkeit zu begreifen (kanalisiert nicht zuletzt durch die Tatsache, dass es dafür keine Abrechnungsziffer gibt!). Mangels entsprechender fachlicher Qualifikation bleibt den Gesundheitsprofis oft „nur“ Empathie - offensichtlich nicht genug, wie der boomende Markt esoterischer Produkte und Dienstleistungen zeigt.

Vor diesem Hintergrund ist es umso erstaunlicher, dass das Thema Spiritualität jetzt zunehmend häufig in den Fokus medizinischer Diskussionen gelangt. Naheliegend, dass eine besondere Affinität der palliativen Medizin zur Spiritualität besteht, also dann, wenn unheilbar kranke Menschen in Krankenhäusern und Hospizen in den letzten Schritten ihres Lebenswegs begleitet werden.

Wer aber hätte erwartet, dass $71 \%$ aller Menschen, die in einer Akutklinik aufgenommen wurden, einen „bedeutsamen spirituellen Glauben“ angaben, auch wenn dies häufig nicht in religiöser Form ausgedrückt wurde ${ }^{1}$. Auch für die klinische Perspektive ist die Spiritualität offensichtlich ein wichtiges Kriterium. So ist die Frage „ob“ wohl inzwischen obsolet, es geht um die differenzierte Betrachtung des „was“ und des „wie“, wie es vor kurzem ein Editorial im renommierten British Medical Journal diskutierte (Titel: „Spiritual needs in health care“2).

Die Osteopathie, für die spirituelle Aspekte schon seit Still immer integraler Bestandteil waren, könnte diese Entwicklungen müde lächelnd verfolgen. Sollte sie aber nicht. Vielmehr sollte die Bestätigung, es „schon immer gewusst“ zu haben, eine besondere Herausforderung darstellen, auch und gerade diese Potenziale stets und immer wieder aufs Neue bewusst zu mobilisieren und einzubringen in die Therapie.

\section{Die Herausgeber}

\section{Literatur \\ Psychol Med. 2001;31:1015-23 \\ 2 Brit Med J. 2004:329:123-4}

